\title{
The Magnetopause Energetic Electron Layer 1. Observations Along the Distant Magnetotail
}

\author{
D. N. BAKeR ${ }^{1}$ and E. C. Stone \\ California Institute of Technology, Pasadena, California 91125
}

\begin{abstract}
Observational features of the magnetopause energetic electron layer have been studied along the distant magnetotail $\left(-10 R_{E} \gtrsim X_{\mathrm{SM}} \gtrsim-40 R_{E}\right)$ using California Institute of Technology instrumentation aboard Imp 8. On the basis of 101 layer crossings obtained on 60 spacecraft orbits it is concluded that the layer of electrons $(E \gtrsim 200 \mathrm{keV}$ ) was present on $97 \%$ of the magnetopause crossings during 1973-1975. The inner edge of the layer is typically found to correspond to the magnetopause boundary as determined from concurrent plasma analyzer and magnetometer data. Energetic electrons within the layer are observed to be persistently streaming tailward along local magnetosheath field lines. Pitch angles of electrons along the dusk magnetopause are often restricted to values of $\leqslant 40^{\circ}$, whereas along the dawnside of the tail somewhat larger pitch angles are normally seen. Differential energy spectra of electrons in the layer are typically fit by power law forms with spectral indices $2 \lesssim \gamma \leqslant 4$, and intensity enhancements often extend to energies of $\gtrsim 1 \mathrm{MeV}$. Average directional intensities $(E \gtrsim 200 \mathrm{keV})$ flowing through the layer are remarkably constant, ranging from $\sim 1$ to $\sim 5\left(\mathrm{~cm}^{-2} \mathrm{~s}^{-1}\right)$, but apparent 'hot spots' are found along the dawn flank of the magnetotail. It is suggested that these hot spots are near a source region for energetic electrons.
\end{abstract}

\section{INTRODUCTION}

Energetic electrons near the magnetopause have been reported by a number of authors (see, for example, Frank and Van Allen [1964] and Singer and Bame [1970]). That energetic electrons $(E>40 \mathrm{keV})$ form a layer near the magnetopause was first suggested by $M$ eng and Anderson [1970]. The energetic electron layer was found to be $1-2 R_{E}$ in thickness for regions generally sunward of the dusk-dawn meridian plane and was found to be persistently present near the mid-to-highlatitude magnetopause. Though less detailed information was given, the data of Meng and Anderson also suggested that the electron layer was present at $\sim 60 R_{E}$ along the flanks of the magnetotail.

Heos 2 data [Page et al., 1973; Domingo et al., 1974, 1977] subsequently showed both that the energetic electron layer was present in regions nearly directly above the north geographic pole and that frequently the electrons within the layer had energies of as much as 1 or $2 \mathrm{MeV}$. Domingo et al. [1977] found evidence for $\lesssim 10 \%$ net tailward flow of the electrons.

$M$ eng and Anderson [1975] presented electron data ( $E>18$ $\mathrm{keV}$ to $E>120 \mathrm{keV}$ ) at high latitudes near the dayside magnetopause and within the polar cusp region. It was again found that the magnetopause layer was persistently present. The integral electron energy spectrum was found to typically be fit by a hard power law distribution $\left(J \propto E^{-2}\right)$, and the electrons ( $E>45 \mathrm{keV}$ ) were characterized as having nearly isotropic pitch angle distributions.

In prior reports [Baker and Stone, $1977 b, c]$ we described observations made in the vicinity of the tailward magnetopause, about the entire circumference of the tail. Evidence was presented in those brief papers for a persistent layer of $\gtrsim 200$ $\mathrm{keV}$ electrons within several earth radii of the magnetopause along the distant magnetotail. Significant net tailward flow of electrons with energies of $\gtrsim 200 \mathrm{keV}$ was observed corresponding to a large $\left(\gtrsim 10^{14} \mathrm{ergs}^{-1}\right)$, nearly continual energy flow along the downstream magnetopause. In this paper we present further details about the magnetopause energetic electron

${ }^{1}$ Present address: Los Alamos Scientific Laboratory, Los Alamos, New Mexico 87545.

Copyright $(\mathcal{C} 1978$ by the American Geophysical Union. layer (MPL) using California Institute of Technology (Caltech) data from the time of Imp 8 launch through 1975.

\section{INSTRUMENTATION AND DATA ANALYSIS}

Imp 8 was launched on October 25,1973 , into a mildly elliptical ( $23 R_{E}$ by $46 R_{E}$ ) earth orbit with initial inclination to the ecliptic of $29^{\circ}$ and an orbital period of 12.2 days. Hence during the course of a typical year of observation the spacecraft covers a relatively large portion of the cislunar magnetotail from high southerly latitudes to high northerly latitudes. During certain 'seasons' the spacecraft passes through the midmagnetotail at low latitudes $\left(\theta_{\mathrm{SM}} \sim 0^{\circ}\right)$ and samples the plasma sheet extensively. Of additional considerable interest here, however, are magnetotail passes during which Imp 8 reached high solar magnetospheric latitudes and sampled the high tail lobe-magnetosheath interface.

The Caltech electron/isotope spectrometer (EIS) aboard Imp 8 measures electrons $(E>160 \mathrm{keV})$ and protons $(E>1.3$ $\mathrm{MeV}$ ), as well as heavier nuclei. The instrument provides both counting rate data at several different (essentially integral) threshold energies and differential energy spectra in a pulse height analysis mode ( $\sim 40-\mathrm{keV}$ energy resolution). Pulse height and counting rate data are all accumulated by an onboard system into eight sun-fixed $45^{\circ}$ sectors. The spacecraft spins with its rotational axis nearly perpendicular to the ecliptic plane (spin period of $\sim 2.6 \mathrm{~s}$ ), and the EIS viewing aperture is perpendicular to the spacecraft spin axis (i.e., parallel to the ecliptic plane)

Temporal resolution for counting rates is available on scales as short as $\sim 5 \mathrm{~s}$. However, angular distributions are formed during seven consecutive spacecraft rotations and hence comprise $\sim 20$ s of data. We ordinarily average a number of samples together to form longer temporal averages when they are appropriate or when statistical considerations require such averaging.

A second-order Fourier least-squares fit is made to the angular distribution data. The assumed analytic form is

$$
C(\phi)=C_{0}\left[1+S \cos \left(\phi-\Delta_{1}\right)+K \cos \left(2 \phi-2 \Delta_{2}\right)\right]
$$

Here $C$ is the number of counts observed in the azimuthal $(\phi)$ direction and $C_{0}$ is the spin-averaged number of counts. $S$ and 

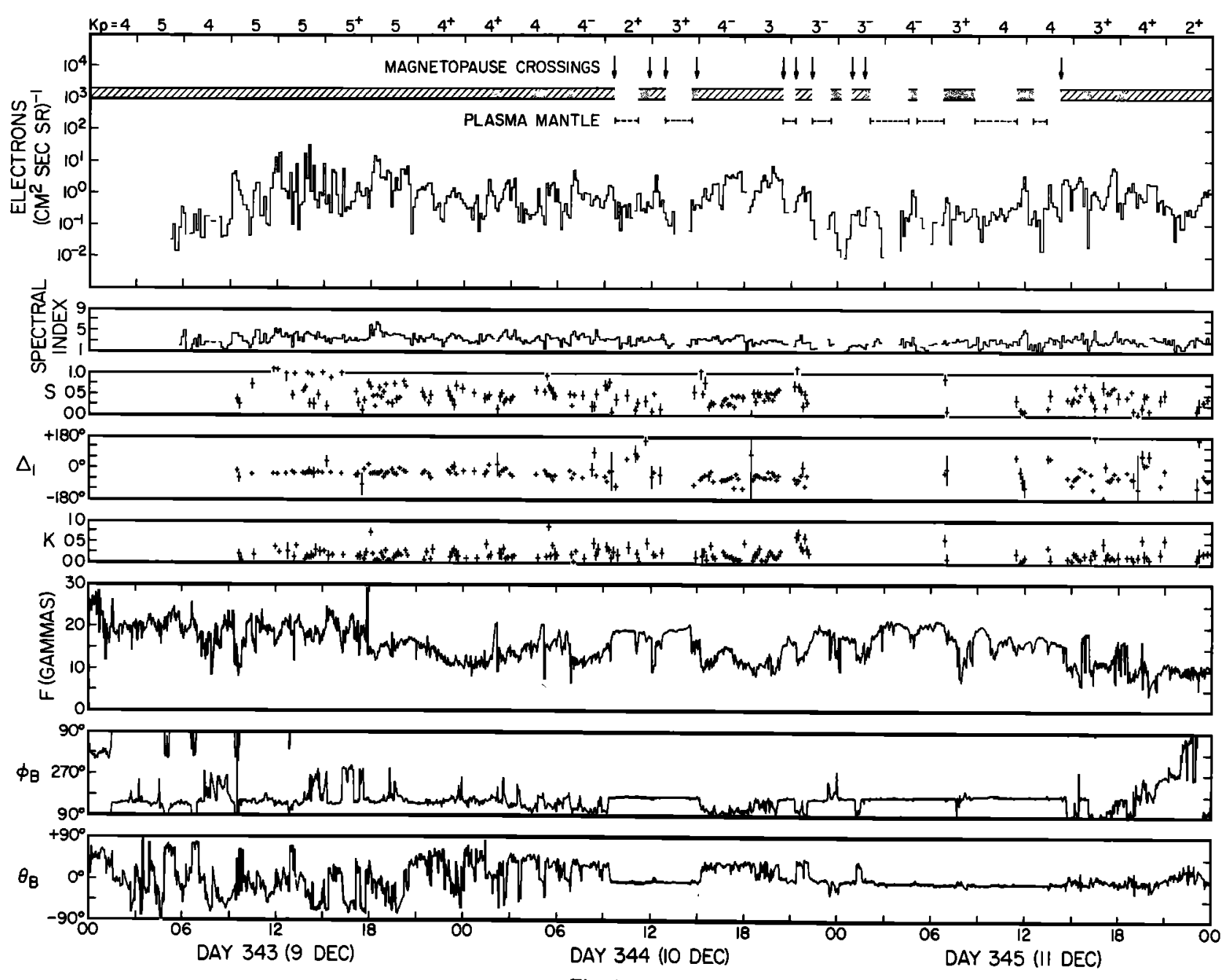

Fig. $1 a$

Fig. 1. Detailed particle data and magnetic field data for the period December 9-14, 1974. The plasma flow data are summarized in the upper panel by the horizontal shaded bar, the shading having the same meaning as in Figure 3 . Also shown in the upper panel are 8.2-min averages of fluxes of $\gtrsim 200-\mathrm{keV}$ electrons, and at the top are the 3-hour $K p$ values. In descending order are also shown the power law differential energy spectral index (second panel), the streaming amplitude for $\gtrsim 200-\mathrm{keV}$ electrons ( $S$, third panel), the detected direction of maximum electron streaming in solar ecliptic longitude $\left(\Delta_{1}\right.$, fourth panel), and the second-order modulation amplitude ( $K$, fifth panel). The bottom three panels show the concurrently measured magnetic field data (122.7-s averages) obtained with Imp 8 (courtesy of $R$. P. Lepping and N. F. Ness), including the total field strength $F$, the solar magnetospheric (SM) field azimuth $\phi_{B}$, and the SM field latitude $\theta_{B}$. Hours in universal time are shown along the bottom: (a) December 9-11, 1974, and (b) December 12-14, 1974. A background of $9 \times 10^{-2} \mathrm{~cm}^{-2} \mathrm{~s}^{-1} \mathrm{sr}^{-1}$ has been subtracted from the electron fluxes.

$K$ are the relative streaming and second-order modulation amplitudes, respectively. The parameters $\Delta_{1}$ and $\Delta_{2}$ give the phase angle in the ecliptic plane $\left(0^{\circ}\right.$ is sunward) at which the maximum modulation amplitude (streaming or nonstreaming) is detected. Further details about data analysis and the EIS are given by Baker and Stone [1977a].

Information available for the current studies, in addition to energetic particle data from the EIS, includes concurrent plasma analyzer data (L. A. Frank, private communication, 1975) and magnetic field data (R. P. Lepping and N. F. Ness, private communications, 1976, 1977).

The plasma analyzer data $(50 \mathrm{eV}<E<45 \mathrm{keV})$ are obtained from the University of Iowa Lepedea aboard Imp 8 and are in the form of color-coded energy-time spectrograms. Imp 8 bow shock crossings are clearly evident in the proton spectrogram, for example, by the extensive energy-broadening which occurs for the proton distribution as the spacecraft leaves the solar wind and enters the magnetosheath. Likewise, magnetopause crossings are evident in the proton spectrograms by the cessation of the extremely intense antisunwarddirected magnetosheath bulk flow of the protons. Within the high lobes of the magnetotail (far inside the magnetopause) the plasma spectrograms show only a very low background response.

The magnetic field data from the Goddard Space Flight Center fluxgate magnetometer are provided in the form of 15.36-s average vectors in both solar ecliptic (SE) and solar magnetospheric (SM) coordinates. Vector averages of these data have been performed when longer time intervals are appropriate. Bow shock crossings are quite evident in the magnetic field data as the field strength increases abruptly in magnitude from a fairly constant interplanetary value $(\sim 5 \times$ $10^{-5} \mathrm{G}=5 \gamma$ ) to a highly fluctuating $10-20 \gamma$ within the magnetosheath. The field direction in the magnetosheath is also disordered and fluctuating. Magnetopause crossings are evident in the magnetic field data, since the field typically 


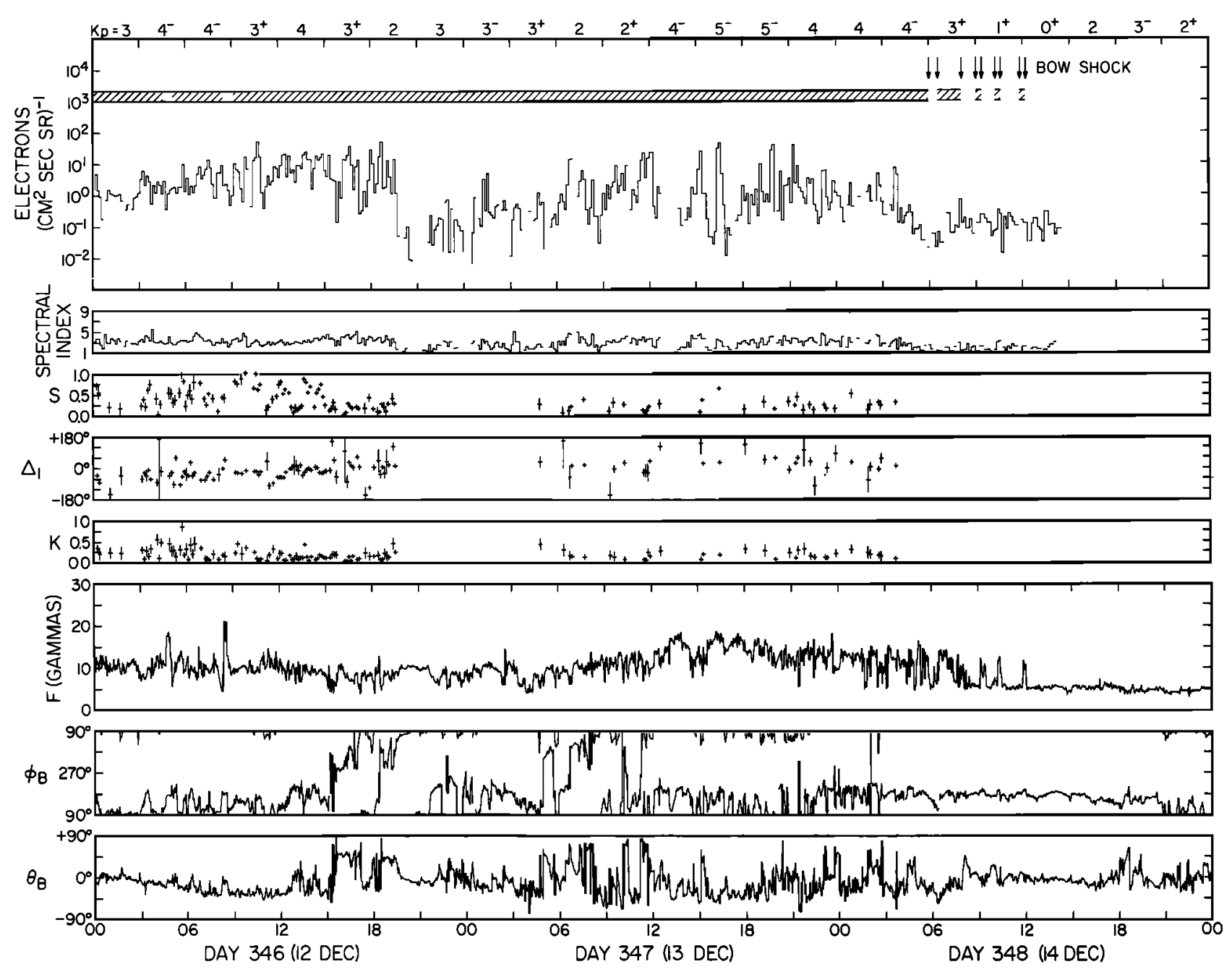

Fig. $1 b$

becomes steady ( $\sim 20 \gamma$ in magnitude) and well ordered (sunward and antisunward) as the spacecraft goes from the magnetosheath into the high lobes of the tail.

Comparison of the magnetic field data and the plasma analyzer data used here shows the general compatibility of the boundary (bow shock or magnetopause) identifications made using either the magnetic field or the plasma criterion. Some ambiguity occasionally exists as to the precise magnetopause location using either plasma data or magnetic field data alone. However, having both data sets available tends to improve the magnetopause identification capability, and these data provide the boundary locations with adequate accuracy for the purposes of this study.

\section{Observations at the Magnetopause}

\section{a. Electron Flux Enhancements and Anisotropies}

In a few fortuitous orbits each year, Imp 8 moves through the earth's magnetotail at very high latitudes and just grazes the magnetopause surface. One such 'skimming' orbit was discussed by Baker and Stone [1977b]. In Figure 1 we give a detailed presentation of energetic particle, plasma, and magnetic field data for this period. The upper panel of the figure summarizes the 8.2-min average fluxes of electrons with $E \gtrsim$ $200 \mathrm{keV}$. The second panel from the top gives a differential spectral index computed from the ratio $j(E \gtrsim 1 \mathrm{MeV}) / j(E \gtrsim$
$200 \mathrm{keV}$ ). The spectral index $\gamma$ assumes a power law distribution $\left(d j / d E \propto E^{-\gamma}\right)$. The third and fifth panels of Figure 1 give the computed values of $S$ and $K$, respectively, for electrons with $E \gtrsim 200 \mathrm{keV}$ (see (1) above). The fourth panel shows the computed direction of the maximum streaming amplitude with $\Delta_{1}$ plotted in the solar ecliptic frame. Hence $\Delta_{1}=0^{\circ}$ implies streaming from the sunward direction (i.e., with tailward flow). Harmonic fits to the observed angular distributions are generally made only when the flux is $\gtrsim 0.5\left(\mathrm{~cm}^{2} \mathrm{~s} \mathrm{sr}\right)^{-1}$, since below this level, counting rates in the various sectors are usually small with large statistical fluctuations.

In the upper panel of Figure 1 the shaded horizontal bar indicates the plasma domains in which the spacecraft was located during the course of this tail passage. The bow shock was observed in the plasma data at $\sim 2130$ UT of day 342 (i.e., prior to the start of Figure 1), and from that time until midday of day 348 the spacecraft was largely within the region of intense tailward magnetosheath ion flow. Relatively brief cessations of this strong flow occurred intermittently on days 344 , 345 , and 346 . These cessations of the strictly magnetosheathlike flow are taken to represent magnetopause crossings at the points indicated in the figure. Thus gaps in the horizontal bar indicate times when Imp 8 was within the high tail (southern) lobe. A mbiguous plasma flow conditions are shown by the dotted shading.

It was often seen on this pass that much-reduced plasma 

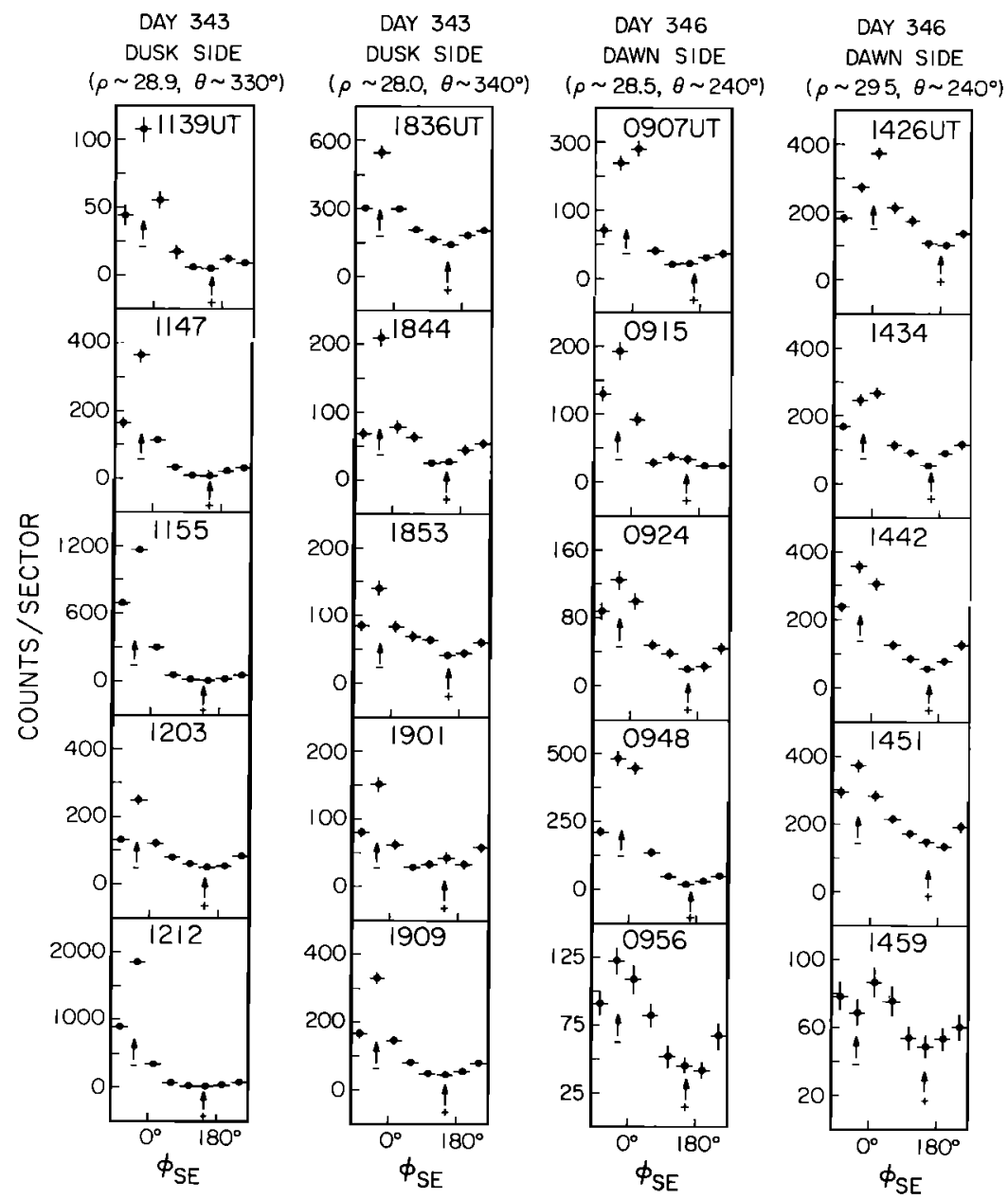

Fig. 2. Sequences of angular distributions of electrons $\gtrsim 200 \mathrm{keV}$ observed during 8.2 -min intervals following the times shown with each sample. The data plotted consist of the observed number of counts/sector during the interval versus the solar ecliptic azimuth $\phi_{\mathrm{SE}}$. The magnetic field direction as projected upon the ecliptic plane $\left(\phi_{B}\right)$ is shown by the positive vertical arrow, while $\phi_{\theta}-180^{\circ}$ is shown by the negative arrow. Statistical error bars $( \pm 1 \sigma)$ are shown when they exceed the size of the plotted point. At the top of each panel, $\rho$ and $\theta$ give the spacecraft location as described in the text.

flows, which we interpret as plasma mantle observations, were present inside the magnetopause boundary. The occasions when such conditions were encountered are shown by the labeled dashed lines in the top panel of Figure 1. It is seen that the mantle (i.e., 'boundary layer') plasmas were present at nearly all times, on this particular orbit, when the spacecraft passed within the magnetopause surface.

The magnetic field data are shown in the form of 2-min (122.7 s) averages in the bottom three panels of Figure 1. The magnetic field variations were often quite complex, but several points may be noted immediately. It is seen that the value of $\phi_{B}$ was rather constant on days 343 and 344 along the dusk magnetopause surface. We further note that $\phi_{B}$ was, in this case, typically $135^{\circ}-150^{\circ}$. Hence the field lines draped over the magnetopause were at roughly the 'garden hose angle' of the solar wind, the IMF sector being such that field lines were in the positive or 'away' from the sun configuration.

The signature of magnetopause crossings may be readily recognized in the magnetic field data as, especially, at $\sim 0930$ UT of day 344 at which time $F$ increased to $-20 \gamma, \phi_{B}$ increased to $\sim 180^{\circ}$ as appropriate to the southern tail lobe, and $\theta_{B}$ became relatively steady in value near $0^{\circ}$. (Some brief magnetopause crossings, or near approaches, also appeared to have occurred earlier at $\sim 0200$ and $\sim 0500$ UT of day 344 .) The subsequent switching back and forth from magnetosheath to high-lobe magnetic field conditions may be seen several times in the magnetometer data between $\sim 1500$ UT of day 344 and $\sim 0900$ UT of day 346 . The magnetic field and plasma data are seen to be in general accord with regard to boundary crossings.

The general level of geomagnetic activity is indicated in Figure 1 by 3-hour $K p$ indices shown along the top panel of the figure.

The energetic electron intensities shown in the upper panel of Figure 1 reveal that from $\sim 0900$ UT of day 343 onward there were pronounced flux enhancements for electrons with $E$ $\gtrsim 200 \mathrm{keV}$ (for clarity we omit the $\gtrsim 1-\mathrm{MeV}$ intensity profile, which also showed flux enhancements). Many of the brief incursions through the magnetopause surface to positions within the magnetotail, as during the 1300-1400 UT interval of day 344 , were accompanied by substantial flux reductions, often to near-background levels. Also, the spacecraft was largely within the high tail lobe and/or the plasma mantle during much of the first half of day 345 , and it is specifically noted that during such periods $\gtrsim 200-\mathrm{keV}$ electron fluxes were very low $\left(\lesssim 10^{-1} \mathrm{~cm}^{-2} \mathrm{~s}^{-1} \mathrm{sr}^{-1}\right)$. 
Moderately high fluxes $\left(0.5-10 \mathrm{~cm}^{-2} \mathrm{~s}^{-1} \mathrm{sr}^{-1}\right)$ were observed from $\sim 1500$ UT on day 345 until $\sim 2000$ UT of day 346 , at which time intensities fell to $\sim 10^{-2} \mathrm{~cm}^{-2} \mathrm{~s}^{-1} \mathrm{sr}^{-1}$. Subsequently, the large number of sporadic electron bursts observed on days 347 and 348 may have represented further brief encounters with the outer edge of the MPL or observation of the sporadic intensity spikes often associated with the bow shock.

In the third panel from the top of Figure 1 it is seen that ordinarily when the spacecraft was outside the magnetopause the degree of unidirectional streaming was large. On many occasions, the computed value of $S$ was $\sim 1.0$, implying that the front-to-back intensity ratio was of the order of 100:1 averaged over an 8.2 -min period. It is also seen in the fifth panel of Figure 1 that ordinarily the second-order modulation amplitude $(K)$ was small compared with $S$. A few exceptions to this may be seen to occur, usually very near magnetopause crossings (cf. data at $\sim 2200$ UT of day 344 when $K$ was large and comparable with $S$ ).

Comparison of the value of $\Delta_{1}$ (fourth panel of Figure 1) with $\phi_{B}$ shows that the electron streaming was generally field aligned. This is more readily evident in Figure 2, in which are shown several detailed examples of observed $200-\mathrm{keV}$ electron angular distributions for days 343 and 346 . In each specific distribution we have plotted the number of counts per $45^{\circ}$ sector accumulated during a given $8.2-\mathrm{min}$ interval versus the solar ecliptic longitude $\left(\phi_{\mathrm{SE}}\right)$ of the sector. Each example also shows the locally measured magnetic field azimuthal direction $\phi_{B}$ by the short vertical arrow (labeled ' + '), while $\phi_{B}-180^{\circ}$ is also shown (labeled '-').

Many of the distributions shown in Figure 2 appear to be very sharply peaked along the field line direction. This tendency is particularly clear along the dusk magnetopause. An analysis of the true, deconvolved electron pitch angle distributions within the MPL has been performed as described below. We have detailed laboratory calibrations of the angular response of the EIS to a unidirectional beam of energetic electrons. By numerically convolving the calibrated detector angular response function with various assumed 'beam profiles' we arrive at the expected detector response as a function of scan direction (relative to the beam direction). In such analyses we have used a number of assumed beam profiles, such as the following: rectangular, wherein the beam flux $S(\alpha)$ is isotropic for pitch angles from $\alpha=0^{\circ}$ to $\alpha=\alpha_{0}$ and $S(\alpha)=0$ for $\alpha>$ $\alpha_{0}$; Gaussian, $S(\alpha)=\exp \left(-\frac{1}{2} \alpha^{2} / \alpha_{0}^{2}\right)$; parabolic, $S(\alpha)=1-$ $\left(\alpha / \alpha_{0}\right)^{2}$ for $\alpha \leq \alpha_{0}, S(\alpha)=0$ for $\alpha>\alpha_{0}$; and cosinusoidal, $S(\alpha)$ $=\cos \left(\pi / 2 \cdot \alpha / \alpha_{0}\right)$ for $\alpha \leq \alpha_{0}, S(\alpha)=0$ for $\alpha>\alpha_{0}$. Thus for a wide range of $\alpha_{0}$ values we can simulate EIS responses to beams of various cross sections ranging from nearly unidirectional to isotropic.

Since we are constrained in our ultimate angular resolution by the fact that our data are sectored by the on-board system, we are able to obtain only a rough idea of the true beam profile. In fact, with distributions such as those seen in Figure 2 , where only three sectors show appreciable flux enhancements above background, information about the beam may be completely characterized by the counting rate in the peak sector $(B)$ and the ratios of the counting rates $(A$ and $C)$ in the sectors adjacent to $B$. Thus in this sense, $B$ characterizes the beam intensity, while $A / B$ and $C / B$ characterize the beam width $\alpha_{0}$.

Since the ratios $A / B$ and $C / B$ depend on the form of the beam profile (rectangular, Gaussian, etc.) as well as on $\alpha_{0}$, a given set of ratios is not unique. However, relatively narrow beams $\left(\alpha \leqslant 50^{\circ}\right)$ with rectangular, parabolic, and cosinusoidal beam profiles produce rather similar $A / B$ and $C / B$ ratios for the EIS detectors, while the Gaussian distribution, with its very broad tail, produces quite a distinct expected response. The rectangular beam profile might be expected to result, for example, if an isotropic electron population were produced in a region of relatively high field strength $\left(B_{M}\right)$ and this population then moved into a region local to Imp 8 in which the field strength $\left(B_{L}\right)$ was much lower. The rectangular profile would result from this 'magnetic focusing' if the electron motion were adiabatic, without significant scattering. The adiabatic motion would result in the following relationship:

$$
\sin \alpha_{0}=\left(B_{L} / B_{M}\right)^{1 / 2}
$$

Making the rectangular beam profile assumption and analyzing all periods on day 343 , for example, we find that typically $\alpha_{0} \sim 40^{\circ}$; i.e., the distributions are generally consistent with particles having $\alpha<40^{\circ}$. As seen in Figure 1, the field strength on day 343 is variable, but typically $B_{L} \sim 15 \gamma$. Hence, under these conditions, (2) implies $B_{M} \sim 35 \gamma$. If magnetic focusing produced the highly beamed population observed along the dusk magnetopause, then field strengths in an isotropic source region would be $\sim 35 \gamma$; we note that such values of $B$ are typical near the low-latitude dayside magnetopause [e.g., Behannon, 1968] and near the polar magnetopause [e.g., Fairfield, 1976].

A Gaussian beam profile might also result from the magnetic focusing, with the additional feature of significant scattering of particles. For the day 343 period, for example, the assumption of a Gaussian beam profile gives a typical value of $\alpha_{0} \sim 23^{\circ}$. (Note that the half width at half maximum of such a beam would be $\sim 27^{\circ}$ as compared with the rectangular half width of $\sim 40^{\circ}$.)

The dawnside distributions, such as those shown for day 346 in Figure 2, tend to be broader, less highly beamed. Assuming a rectangular beam profile in the analysis of all pitch angle distributions on day 346, for example, gives $\alpha_{0} \sim 50^{\circ}$. This typical maximum pitch angle is therefore $\sim 10^{\circ}$ greater than that on the dusk side. (For a Gaussian beam profile, $\alpha_{0} \sim 37^{\circ}$ on day 346.) Such differences of beam width between the dusk and the dawn MPL are often seen.

The magnetic field azimuthal direction shown in each example of Figure 2 shows the field-aligned character of the electron streaming. Note that a beam of particles moving along a field line which is at a large angle to the $\operatorname{Imp} 8$ spin plane may be outside of the EIS acceptance cone. This in turn can produce some of the variability in the flux time profiles, such as those seen on day 343 (cf. Figure 1) when $\theta_{B}$ was variable and often large.

Extended periods of essentially continuous electron intensity enhancement near the magnetopause of the type shown in Figure 1 and the persistent large-amplitude streaming conditions have been seen in several other Imp 8 skimming passes. More typically, the spacecraft trajectory produces one rather brief inbound cut and one similarly brief outbound cut through the MPL. For example, Figure 3 shows data acquired with Imp 8 on November 26-30 (days 330-334), 1974. Note in the $Y-Z$ plane projection of the upper portion of Figure 3 that this pass was clearly not of the skimming variety. At the very bottom of Figure 3 are summarized the measured absolute intensities of electrons with $E \gtrsim 200 \mathrm{keV}$ for this period. A large electron flux enhancement was seen in the magnetosheath beginning at $\sim 1200 \mathrm{UT}$ of day 330 . The energetic 

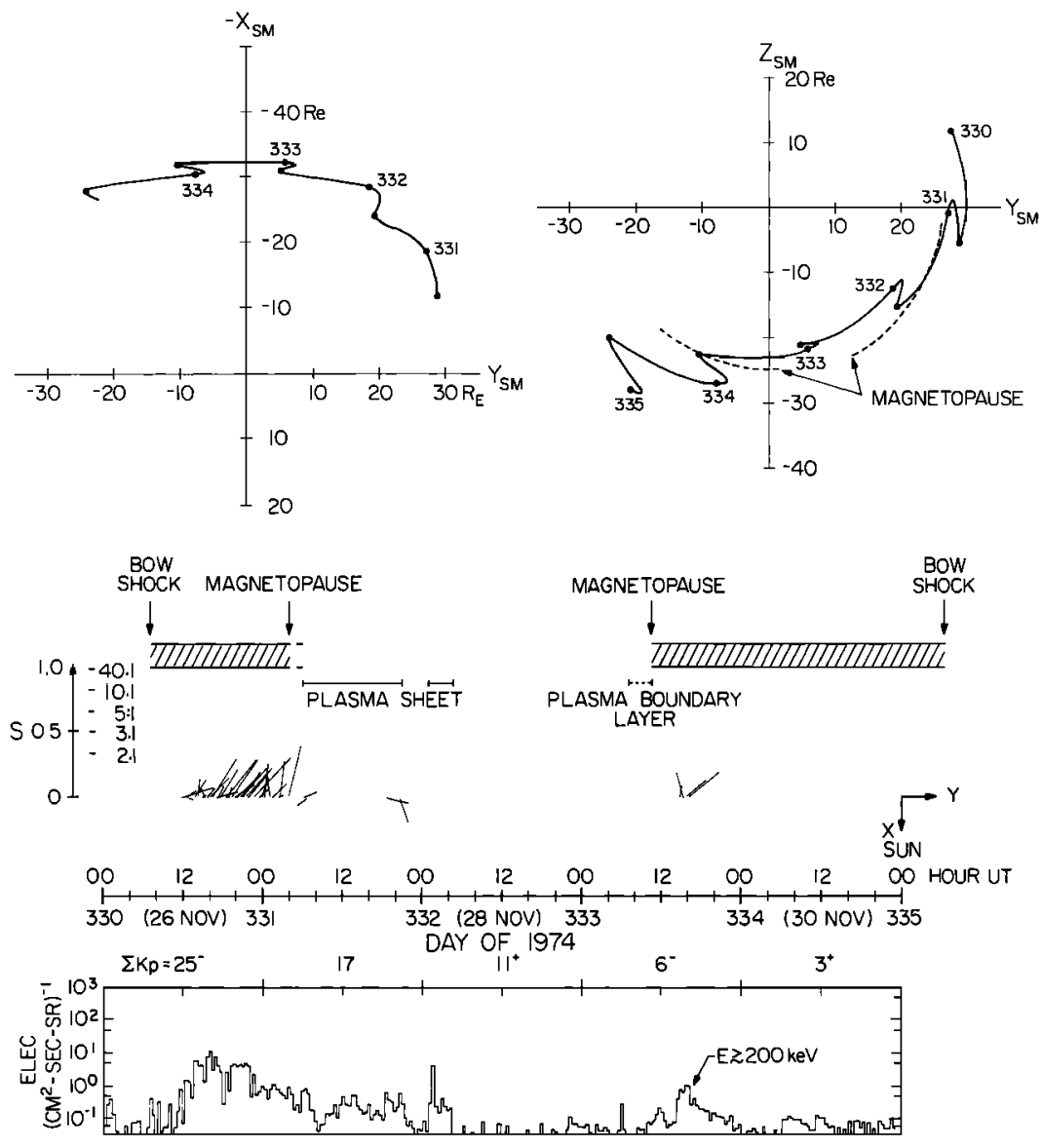

Fig. 3. Imp 8 trajectory (upper portion of the figure) plotted in the geocentric solar magnetospheric coordinate system with solid circles spaced at 12 -hour intervals. The observed flux time profile of electrons with $E \gtrsim 200 \mathrm{keV}$ (based on 24.6min averages) is shown by the lowest panel, above which is shown the daily sum of $K p$. Tailward plasma ion flow of the magnetosheath is shown by the cross-hatched horizontal bar, gaps in the central portion of the bar indicating passage of the spacecraft into the magnetotail. Observations of boundary layer (mantle) plasmas are indicated by the horizontal dashed lines, while ambiguous flow regions are shown by dotted shading. The flow direction and relative streaming amplitude of $\geq 200$-keV electrons are shown by the anisotropy vectors in the central part of the figure for intervals during which the spinaveraged flux was $\geq 0.5 \mathrm{~cm}^{-2} \mathrm{~s}^{-1} \mathrm{sr}^{-1}$.

electron flow vectors in the center of the figure show a wellordered pattern of streaming external to the magnetopause boundary which was crossed at $\sim 0500$ UT of day 331. Lowlevel plasma sheet energetic electron intensity enhancements on days 331 and 332 were nearly isotropic.

Along the top of the intensity panel in Figure 3 we show the level of geomagnetic activity indicated by the daily $K p$ sums, $\sum K p$. It is seen that a large systematic decrease in activity occurred during this period, since $\sum K p$ was $25-$ on day 330 but $\sum K p$ diminished to only $3+$ on day 334 . The MPL was evidently present on the geomagnetically quiet day 333 , but the layer appeared at that time to be of rather low peak intensity and was apparently very thin. Defining $\rho \equiv\left(Y^{2}+Z^{2}\right)^{1 / 2}$, the apparent layer thickness from its outer edge $\left(\rho_{0}\right)$ to its inner edge $\left(\rho_{t}\right)$ on day 333 then was only $\Delta \rho \simeq 1.1 R_{E}$.

Other passes through the MPL have been examined in detail in order to identify any differences between the northern and southern MPL. No obvious asymmetries were found in absolute electron intensity, relative anisotropy amplitude, or average direction of particle flow.

Figure 4, taken from Baker and Stone [1977c], represents a set of complete MPL crossings made during 1973, 1974, and 1975 observed with Imp 8 EIS. Portions of the Imp 8 trajectory (as projected upon the $Y_{\mathrm{SM}}-Z_{\mathrm{SM}}$ plane) during which enhanced intensities and energetic electron flows were observed have been made visible. Of all the spacecraft magnetotail passages during the 1973-1975 period, a number of cuts through the magnetopause were unusable for present purposes due either to data gaps or to high solar flare ambient intensities. In addition, on three cuts through the magnetopause region (during which there were no data gaps) we found essentially no intensity enhancements (above the interplanetary level) in the $\gtrsim 200-\mathrm{keV}$ electrons. Hence in these cases, which occurred at geomagnetically quiet periods, the MPL was apparently absent, at least in the high-energy range. The remaining useful cases from 60 spacecraft orbits appear in Figure 4 and correspond to a total of 101 separate MPL encounters.

The Imp 8 orbit is such that the spacecraft moved to largest $X_{\mathrm{SM}}$ and largest $Z_{\mathrm{SM}}$ values while moving through the southern portion of the magnetotail. This feature appears prominently in Figure 4, since coverage of the MPL extends all the way around the southern portion of the magnetotail. Imp 8 did not 


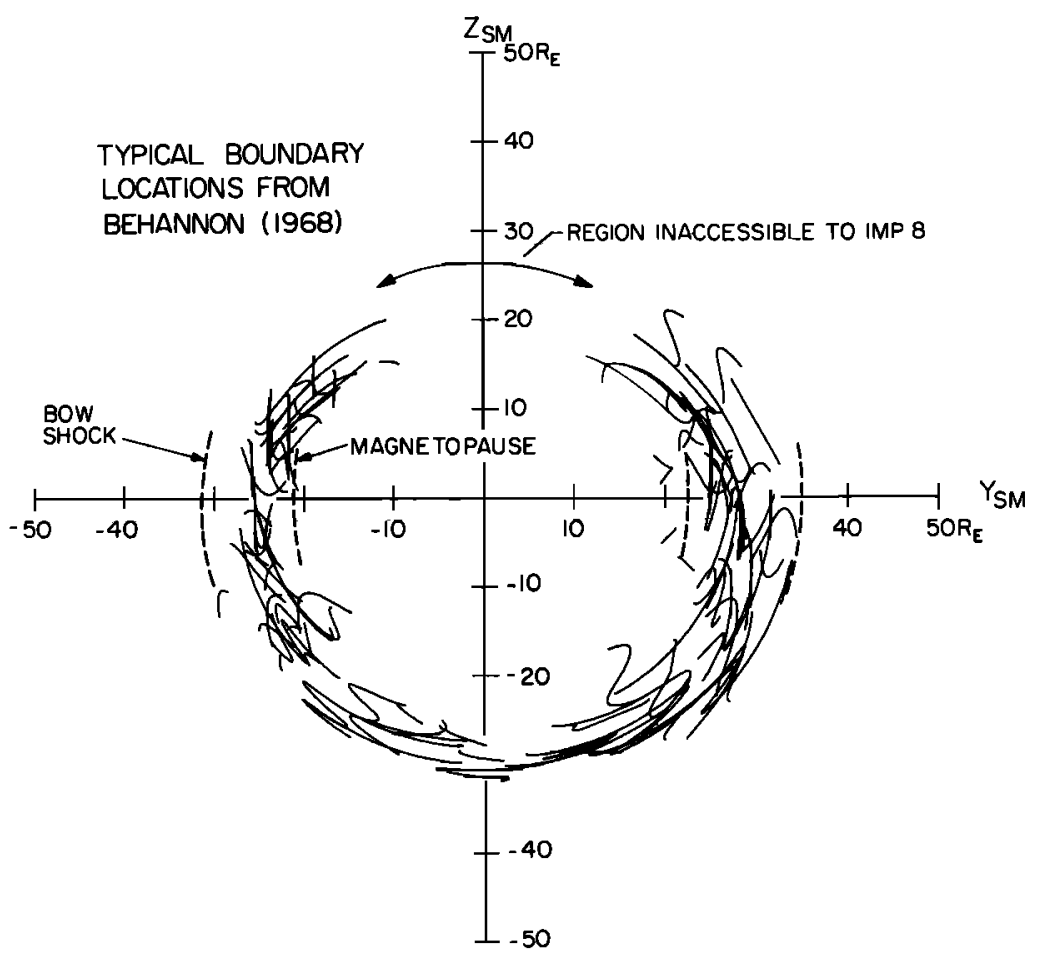

Fig. 4. Set of 101 complete Imp 8 magnetopause layer crossings obtained with the Caltech EIS during 1973, 1974, and 1975. The $Y-Z$ plane projection of the spacecraft trajectory has been made visible for times during which enhanced intensities and fows of the layer were observed. Regions not covered by Imp 8 because of orbital constraints and the typical boundary locations (bow shock and magnetopause) found by Behannon [1968] are shown. Since the spacecraft crosses the MPL at various $X_{\mathrm{SM}}$, this figure is not a planar cross section of the MPL.

reach northern latitudes adequately high to allow it to sample the entire northern layer. It seems reasonable to infer, however, that the MPL extends completely around the magnetotail surface despite the failure of Imp 8 to sample high northern latitudes.

The MPL ordinarily lies immediately adjacent to the magnetopause, as shown in Figure 5. In that figure we have plotted $\left(\rho_{t}-\rho_{\mathrm{MP}}\right)$ versus $\theta_{i}$, where, as was previously defined, $\rho$ is the radial coordinates in the $Y_{\mathrm{SM}}-Z_{\mathrm{SM}}$ plane and $\theta$ is the polar angle from this $Y_{\mathrm{SM}}$ axis. The normalized magnetopause location is indicated by a heavy circle, and the point at which the spacecraft crossed the apparent inner edge of the layer (characterized by $\rho_{l}$ and $\theta_{l}$ ) is then compared with this normalized magnetopause location. Since it is seen that $\left(\rho_{t}-\rho_{\mathrm{MP}}\right)$ is $\sim 0$ for most of the cases shown in Figure 5 , it follows that the inner edge of the layer and the magnetopause are approximately coincident.

The typical magnetopause and bow shock positions found by Behannon [1968] for low latitudes are shown in Figure 4 and serve as a useful reference. However, as is emphasized by Figure 5, in most cases the inner edge of the MPL is the magnetopause as identified by local plasma and magnetic field data.

\section{b. Energy Spectra}

The energy spectra of the electrons within the MPL are an important aspect both for subsequent computation of energy flows and for source identification purposes. As was described in section $3 a$, one method of describing the energy spectrum assumes $d j / d E \propto E^{-\gamma}$, where $\gamma$ is computed from $j(E \gtrsim 1$ $\mathrm{MeV}) / j(E \gtrsim 200 \mathrm{keV})$. This method has been utilized in Figure 1 , for example, and the time profile of the spectral index so derived is shown in the second panel from the top of that figure.

Examination of the time variation of the spectral index in Figure 1 shows that a typical value within the MPL is $\gamma \sim 3$. Extended periods with $\gamma<2$ or $\gamma>4$ are rare.

A more precise and informative analysis of MPL energy spectra uses the pulse height analysis (PHA) data available with the EIS instrument [cf. Baker and Stone. 1977a]. As was noted in section 2, the maximum energy resolution available with the EIS is $\sim 40 \mathrm{keV}$. We use the narrow geometry detection mode (which is insensitive to pulse pileup of electrons of energy $E \leqslant 90 \mathrm{keV}$ ) and restrict our analysis to the energy range $160 \mathrm{keV} \leqslant E \leqslant 2 \mathrm{MeV}$. At higher energies within this range we combine PHA channels to provide energy bins of similar logarithmic widths throughout the entire energy interval.

Figure 6 shows typical differential energy spectra obtained during five different 1974 MPL encounters. Each spectrum is an average for a 15-min interval indicated by the labels above each example, and all analyzed PHA events (i.e., events from all eight directional sectors) are included. Stated differently, these represent 'spin-averaged' energy spectra.

It is typically the case, and these examples demonstrate, that the power law form tends to fit the detailed differential energy spectra quite well. The solid lines drawn through each individual set of data points in Figure 6 represent a best fit in the least 


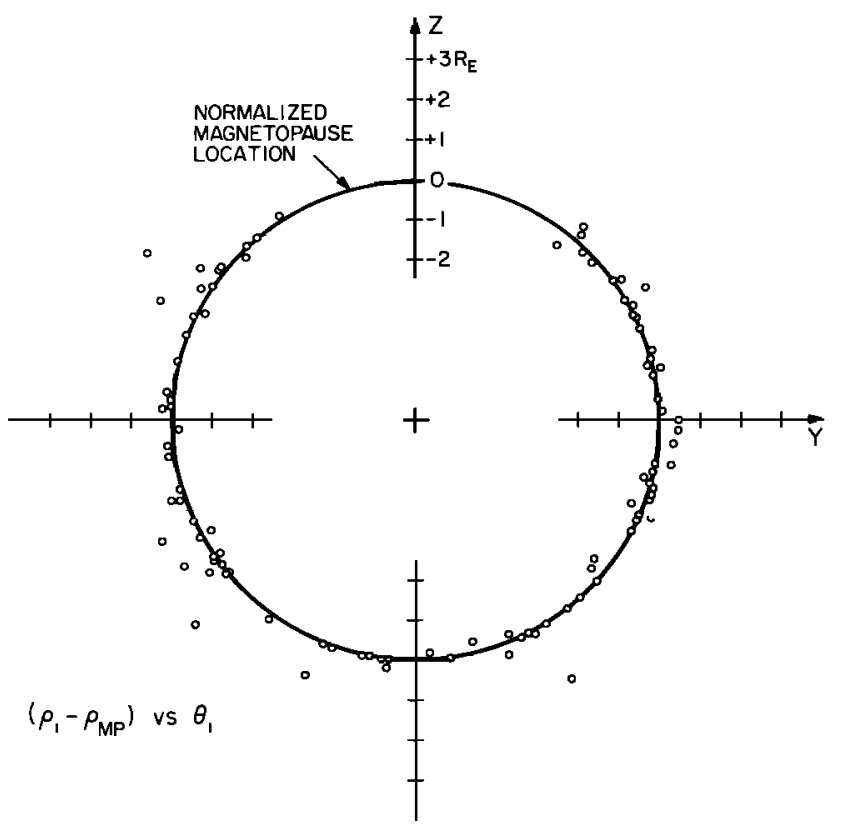

Fig. 5. Observed location (characterized by $\rho_{t}$ and $\theta_{t}$ ) of the inner edge of the MPL as projected on the $Y-Z$ plane, relative to the location of the magnetopause as determined from concurrent plasma and magnetic field data.

squares sense to the PHA data points. The fitted values of $\gamma$ for the examples shown are seen to range from 2.9-3.8.

In the lower portion of Figure 6 we show the values of $K p$

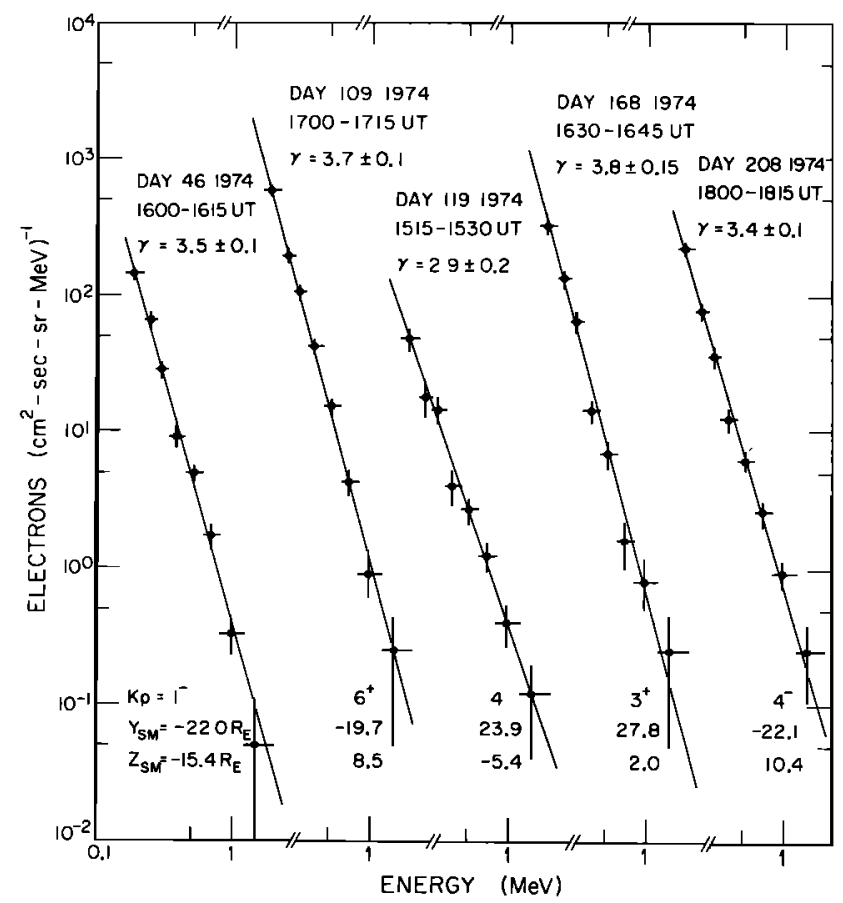

Fig. 6. Typical differential energy spectra for electrons with 160 $\mathrm{keV} \lesssim E \lesssim 2 \mathrm{MeV}$ measured at the indicated times within the magnetopause energetic electron layer. The spectra are obtained from pulse height analysis data, and least squares fits (shown by the solid lines) are made to the flux points to give the best power law distribution in kinetic energy. The spectral indices obtained in the fit are shown in each case, as are the $K p$ value during the observation and the $Y_{\mathrm{SM}}$ and $Z_{\mathrm{SM}}$ position of the spacecraft.

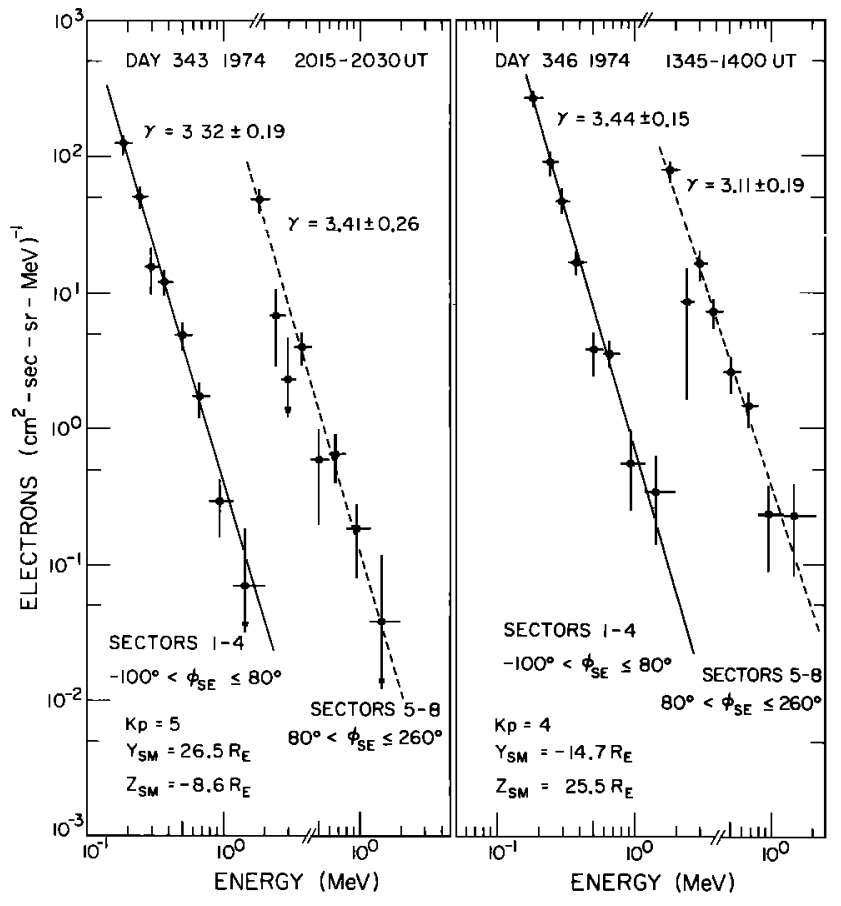

Fig. 7. Same as Figure 6 but where data have now been analyzed in terms of the on-board $45^{\circ}$ sun-fixed sectoring scheme. The front sectors (labeled 1-4) show a hard power law spectrum, such as that seen in Figure 6, while the spectra in the back sectors (5-8) are somewhat irregular.

for the 3-hour period encompassing the observation time, and we also show the spacecraft position in $Y_{\mathrm{SM}}$ and $Z_{\mathrm{SM}}$. The examples are seen to correspond to a wide range of $K p$ conditions from quiet $(1-)$ to highly disturbed $(6+)$ and also to correspond to a wide range of $Z_{\mathrm{SM}}$ positions. Nonetheless, the spectra all appear fairly similar in shape and in absolute intensity.

As was demonstrated, for example, in Figure 2, the $\gtrsim 200$ $\mathrm{keV}$ electrons within MPL ordinarily exhibit a large directional anisotropy. It is therefore of interest to study directional energy spectra in addition to the spin-averaged examples shown in Figure 6. In Figure 7 we show energy spectra which were obtained as follows: Since the beamed energetic electron flux is ordinarily seen at $\phi_{\mathrm{SE}} \sim-30^{\circ}$ (which corresponds to sector 2 of the EIS sectoring scheme), we break up the analyzed PHA events into two directional groups. Sectors 1-4 include detector look angles from $-100^{\circ}$ to $+80^{\circ}$ and thus include the directions of peak particle intensity. Sectors 5-8 $\left(80^{\circ}<\phi_{\mathrm{SE}} \leq 260^{\circ}\right)$ in turn correspond to the back, or downstream, directions in which the minimum directional intensity is always seen.

The examples shown in Figure 7 are typical of the kinds of results obtained when the energy spectra are studied on a directional basis. The front sectors (1-4) for both the duskside (day 343) and the dawnside (day 346) of the tail exhibit the fairly hard power law behavior shown also in Figure 6. The spectra in the back sectors (5-8) show a considerably lower absolute intensity, as expected, but also appear to be poorly fit by a simple spectral form. The dashed lines included with the two 'back sector' spectra in Figure 7 are the formally fitted power law curves.

In Figure 8 we summarize the observed energy spectrum 


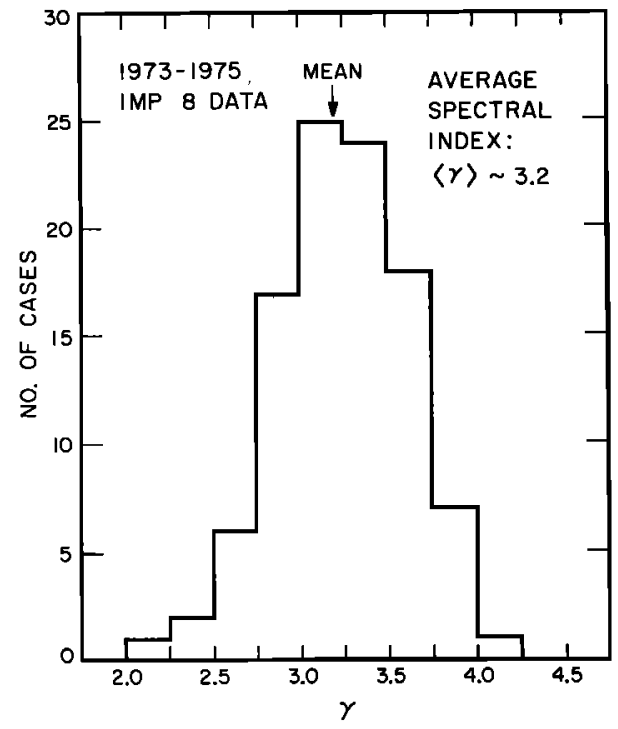

Fig. 8. Frequency of occurrence histogram for the power law spectral index of $\gtrsim 160-\mathrm{keV}$ electrons measured in the peak sector and averaged over each layer crossing. The mean value for all MPL crossings shown is $\langle\gamma\rangle \sim 3.2$.

conditions found in the MPL. For the 101 layer crossings shown in Figure 4, we have computed the average power law spectral index $\gamma$ in the peak sector during each crossing. The histogram showing the distribution of occurrence of $\gamma$ values is then presented in Figure 8. Average indices range from $\sim 2.0$ to $\sim 4.2$, and the mean value for all MPL encounters is $\langle\gamma\rangle \sim 3.2$. In a previous paper [Baker and Stone, 1977c] we have shown that there is a mild but significant $K p$ dependence to the value of $\gamma$ observed within the MPL. This $K p$ trend has not been removed from the frequency of occurrence histogram in Figure 8.

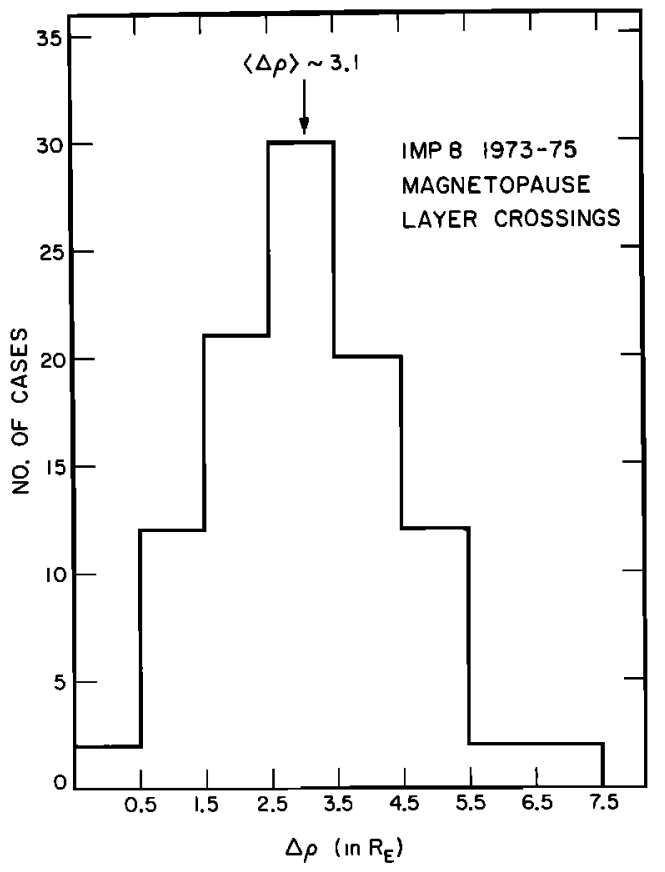

Fig. 9. The frequency of occurrence histogram for the apparent layer thickness $\Delta \rho$ of each of the 101 layer crossings used in this study. The value of $\Delta \rho$ is known to depend on geomagnetic activity, but no such trend has been removed. The mean apparent MPL thickness for all cases is $\langle\Delta \rho\rangle \sim 3.1 R_{E}$

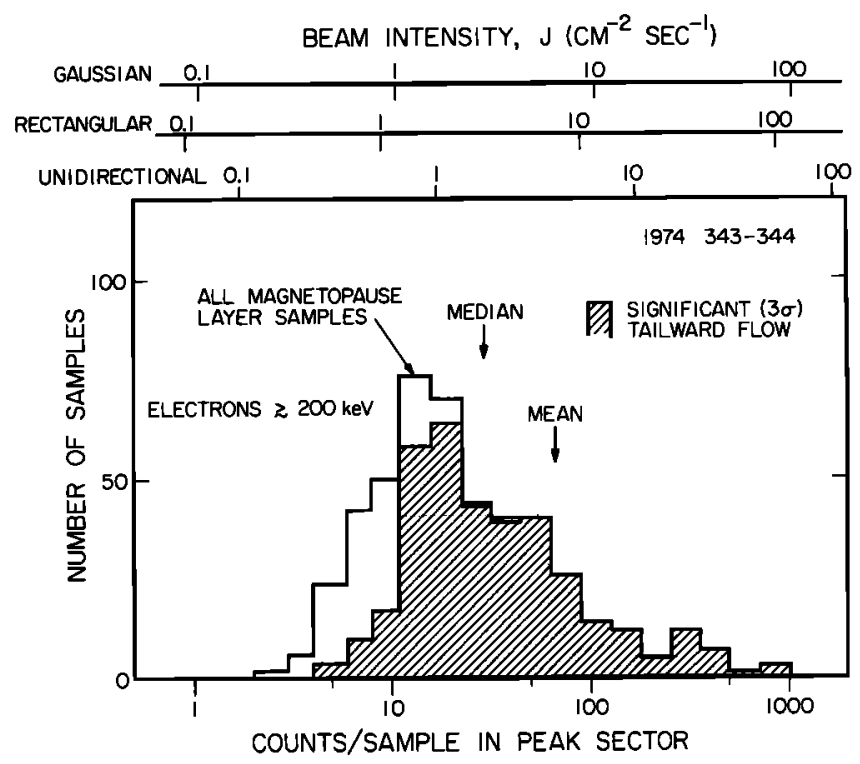

Fig. 10. Histograms illustrating the distribution of the number of samples exhibiting a given number of counts in the peak sector per 4.1min sample for the duskside MPL crossing in Figure 1. The shaded and unshaded histograms, as well as the beam intensity scales, are discussed in the text.

\section{c. MPL Thicknesses and Average Intensities}

Data such as those shown in Figure 4 suggest that the magnetopause electron layer (in the $Y-Z$ plane) has roughly an annular cross section. The annular thickness, given by $\Delta \rho \equiv \rho_{0}$ $-\rho_{t}$, is variable from one layer crossing to the next. We have computed the apparent layer thicknesses for each of the 101 MPL crossings shown in Figure 4, and the frequency of occurrence histogram obtained for these cases is shown in Figure 9. Although the thickness is dependent on geomagnetic activity [Baker and Stone $1977 c]$, no $K p$ trend has been subtracted from the apparent thickness data of Figure 9.

We use the phrase 'apparent layer thickness' because our

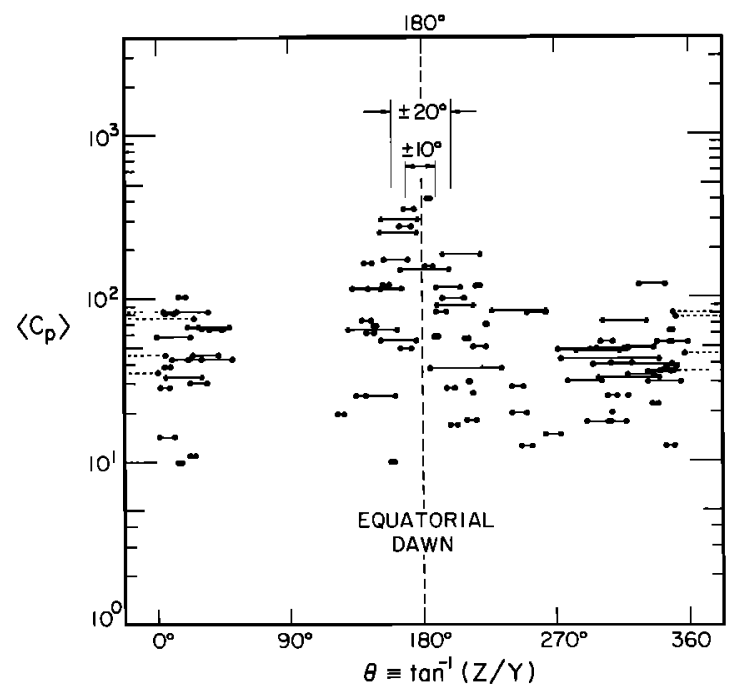

Fig. 11. Mean number of counts per sample in the peak sector, $\left\langle C_{p}\right\rangle$, for each layer crossing plotted versus the polar angles $\theta$ at which Imp 8 crossed the layer. For each MPL encounter, $\theta_{i}$ (where the inner MPL edge was seen) has been connected to $\theta_{0}$ (where the outer MPL edge was encountered). A noticeable relative intensity peak is seen for low-latitude dawn crossings $\left(\theta=180^{\circ} \pm 20^{\circ}\right)$. 
analysis implicitly assumes stationary boundaries (e.g., a stationary magnetopause surface). Clearly, the magnetopause moves considerably on the time scale of several hours, or more, for which Imp 8 was typically within the MPL during each crossing. We presume, however, for the large number of cases used here that the magnetopause was as frequently moving toward the spacecraft as it was moving away and that an approximate average thickness may be discerned. 'Flaring' of the magnetopause as one goes to greater distances down the tail has been considered as possibly affecting our $\Delta \rho$ values. Investigation of this effect has shown, however, that no significant correction is typically required over the range of $X_{\mathrm{SM}}$ values involved in these measurements.

Figure 9 shows that the etectron layer, as measured at points along the distant tail, has typical apparent thicknesses of $1 \lesssim$ $\Delta \rho \lesssim 5 R_{E}$. The mean thickness for all crossings used here was found to be $\langle\Delta \rho\rangle \sim 3.1 R_{E}$.

An additional feature of considerable interest is the average energetic electron intensity measured for each crossing of the MPL. Several aspects related to the question of the $\gtrsim 200-\mathrm{keV}$ electron intensity, for one particular layer passage, are shown in Figure 10. The data in Figure 10 refer to days 343-344, 1974 (i.e., the duskside MPL encounter shown in Figure 1).

Consideration of the sharply peaked angular distributions for electrons such as those shown in Figure 2 indicates that quite often most of the measured intensity occurs in one peak sector. In Figure 10 we have used 4.1 -min data samples and have plotted the frequency of occurrence histogram for the observed number of counts per sample in the peak sector. The unshaded histogram includes samples for all times between $\sim$ 0900 UT of day 343 and 0000 UT of day 345, during which the spacecraft was external to the magnetopause and presumably within the MPL. The shaded histogram in the figure shows the number of cases which exhibited significant tailward field-aligned streaming during this period. The shaded histogram corresponds to the broad range of counts per sample from $\lesssim 10$ to $\sim 1000$.

By using the appropriate accumulation time for one sector during the $4.1-\mathrm{min}$ sample period $(6.8 \mathrm{~s})$ and using the effective area of the EIS for a unidirectional beam of electrons $\left(2.6 \mathrm{~cm}^{2}\right)$ it is possible to convert peak counts to unidirectional beam intensity. This has been done in Figure 10 as shown by the upper horizontal scale and indicates beam intensities ranging from $J \leqslant 1\left(\mathrm{~cm}^{2} \mathrm{~s}\right)^{-1}$ to $J \sim 60\left(\mathrm{~cm}^{2} \mathrm{~s}\right)^{-1}$. Also shown are scales of $J$ appropriate for broader beams such as a rectangular beam (with $\alpha_{0}=40^{\circ}$ ) and a Gaussian beam profile $\left(\alpha_{0}=25^{\circ}\right)$. As was noted in section $3 a$, such assumed beam profiles appear to more nearly reflect the true electron pitch angle distribution on this MPL crossing. Clearly, however, the value of $J$ is not strongly dependent upon the beam profile assumption.

The median and mean values of the shaded histogram distribution of Figure 10 are indicated in the figure. We call the number of counts per sample in the peak sector $C_{p}$, and it is seen that for days 343-344 the median $C_{p}$ value was $\sim 35$. This corresponds to $J$ of $\sim 2\left(\mathrm{~cm}^{2} \mathrm{~s}\right)^{-1}$. The mean counting rate was found to be $\left\langle C_{p}\right\rangle \sim 70$, which gives $\langle J\rangle \sim 4\left(\mathrm{~cm}^{2} \mathrm{~s}\right)^{-1}$.

Distributions of the type shown in Figure 10 have been obtained for each of the 101 layer crossings used in this study. The distributions differ considerably from one layer crossing to the next, and we characterize this variation by using the mean counts per sample in the peak sector, $\left\langle C_{p}\right\rangle$. In one type of analysis, we have plotted the measured values of $\left\langle C_{p}\right\rangle$ for the several layer crossings versus the parameter $\theta$ defined previously (viz., $\theta \equiv \tan ^{-1}\left(Z_{\mathrm{SM}} / Y_{\mathrm{SM}}\right)$ ). In this analysis we have $\theta_{t}$, the polar angle at which the inner edge of the MPL was observed, connected to $\theta_{0}$, the angle at which the outer edge of the MPL was observed on a given layer passage. This range of polar angle (over which the spacecraft moved through the layer) and the corresponding average $C_{p}$ value for the passage constitute Figure 11.

It will be noted that, for the most part, $20 \leqslant\left\langle C_{p}\right\rangle \leqslant 100$. Several layer crossings with $\left\langle C_{p}\right\rangle \sim 10$ were observed, and these occurred throughout the full range of $\theta$ values. On the other hand, a noticeable 'hot spot' occurs near $\theta=180^{\circ}$. All layer crossings with $\left(C_{p}\right)$ significantly greater than 100 were seen to have $\theta \sim 180^{\circ}$. These higher average intensities in the lowlatitude dawn region correspond to what might be expected in, or near, a source region. Aside from the low-latitude hot spot, the average fluxes in the layer are remarkably constant, a point which is of some relevance to the question of the source of the particles.

Additional information about a given magnetopause layer crossing is contained in a plot such as that shown in Figure 10. Taking the ratio of the total number of samples under the shaded histogram to the total number of samples under the unshaded histogram gives the fraction of samples showing significant streaming for the MPL crossing. For the day 343344 period shown in Figure 10 this ratio is $\sim 0.75$. Thus for that particular MPL encounter the percentage of samples showing streaming, $R$, was $\sim 75 \%$.

The values of $R$ observed for each of the layer crossings used in this study have been computed. It is found that $50 \% \leqq R \leqslant$ $90 \%$ for the $1973-1975$ period. The average for all layer crossings used here was $\langle R\rangle \sim 72 \%$. Thus the degree of net tailward flow was ordinarily large on the basis of the raw data, and no attempt has been made here to correct for systematic effects which tend to reduce $R$, such as a large angle between the magnetic field and the Imp 8 spin plane.

\section{Summaryof ObServations}

Imp 8 traversals of the distant magnetotail $\left(-10 R_{E} \gtrsim X_{\mathrm{SM}}\right.$ $\gtrsim-40 R_{E}$ ) at virtually all relevant solar magnetospheric latitudes have shown a persistent layer of electrons $(E \gtrsim 200 \mathrm{keV})$ near the magnetopause.

This layer is observed to lie outside the magnetopause as defined either by magnetic field or by plasma flow criteria.

It is specifically noted that the detected energetic electrons move on field lines of magnetosheath, rather than magnetotail, character. The magnetopause layer is thus observed to be distinct from the plasma mantle.

The electrons within the layer at these geocentric distances are observed to ordinarily extend in kinetic energy to $>1 \mathrm{MeV}$.

The differential energy spectra of the electrons are typically fit by power laws $(2.0 \leqslant \gamma \leqslant 4.0)$ with a mean value $\langle\gamma\rangle \sim 3.2$.

The electrons within the layer have been observed to be continually present for hours and even up to several days (with suitable Imp 8 orbits) along both the northern and the southern halves of the magnetotail.

The $\gtrsim 200-\mathrm{keV}$ electrons are observed to be persistently streaming with a net directional flow along the local magnetic field lines.

Along the dusk magnetopause (in either the northern or the southern hemisphere) the flow is strong and usually well ordered in direction (toward $\phi_{\mathrm{SE}} \sim 150^{\circ}$ ).

The flow of electrons $(E \geq 200 \mathrm{keV})$ along the dawn magnetopause is usually more variable in character and direction than on the duskside, a fact related to the dawnside magnetic field character. 
Pitch angles of electrons with energies of $\gtrsim 200 \mathrm{keV}$ are typically $\lesssim 40^{\circ}$ along the dusk magnetopause, while broader distributions (with $\alpha$ generally $\lesssim 50^{\circ}$ ) are observed on the dawnside.

The apparent layer thickness varies from $\lesssim 1 R_{E}$ to $\gtrsim 5 R_{E}$ with a mean value $\langle\Delta \rho\rangle \sim 3.2 R_{E}$.

Average directional intensities through the layer are remarkably constant, typically ranging from $\sim 1$ to $\sim 5\left(\mathrm{~cm}^{2} \mathrm{~s}\right)^{-1}$. However, apparent 'hot spots' are found along the dawn flank of the magnetotail which may be near a source region.

On the average, strong energetic electron streaming is observed for $\sim 70 \%$ of the time during each layer crossing.

A substantial dependence of layer thickness and average intensity on geomagnetic activity is observed and was described previously by Baker and Stone [1977c].

\section{Discussion}

The observations presented here establish that an energetic electron layer structure is present along the magnetopause between $X_{\mathrm{SM}} \sim-10 R_{E}$ and $X_{\mathrm{SM}} \sim-40 R_{E}$. The layer appears to completely envelop the magnetotail and has roughly an annular cross section. Furthermore, the layer is virtually always present, although its spatial extent, and the intensity within it, appear to wax and wane in association with geomagnetic activity. Finally, the data presented here demonstrate that electrons with $E \gtrsim 200 \mathrm{keV}$ in the layer are persistently streaming tailward along field lines external to and draped over the magnetopause surface.

The work of Meng and Anderson [1970, 1975] and Domingo et al. $[1974,1977]$ was generally concentrated in regions with $X_{\mathrm{SM}} \gtrsim-10 R_{E}$ (with rather sparse presentation of more tailward data). Neither Meng and Anderson nor Domingo et al were able to perform detailed anisotropy measurements, but the latter authors noted a tendency of a net flow of electrons of up to $10 \%$ in the antisunward direction. This contrasts with present Imp 8 observations which ordinarily show electrons with energies of $\gtrsim 200 \mathrm{keV}$ to be persistently and strongly streaming. We have attributed this difference to the smaller magnetic intensity at $\operatorname{Imp} 8$, which results in a magnetic focusing.

It seems reasonable that far down the tail $\left(X_{\mathrm{SM}} \lesssim-10 R_{E}\right)$ and at high latitudes field lines may reasonably smoothly continue into the interplanetary medium along the duskside of the tail. This provides a natural sink for the energetic electrons, allowing them to continually stream along field lines in the magnetosheath and into the solar wind beyond. Moreover, the continual flux enhancement observed near the magnetopause combined with the persistent streaming demands a nearly continuous source of particles somewhere (generally earthward of the spacecraft) along the field line. The electron flow diagrams presented above suggest a source that is at the nose or dawn flank of the magnetosphere.

As discussed by Frank et al. [1976], observations near the low-latitude flanks of the magnetotail suggest active local acceleration processes there. Frank et al. cite intense plasma heating and strong plasma jetting as evidence of low-latitude local acceleration. In their model, Frank et al. envisage nearly continual field line merging at an $X$ type neutral line in the cool dense magnetosheathlike plasmas near the edge of the distant plasma sheet. Earthward of the reconnection region, $B_{Z}$ is northward, and field lines are closed and move into the plasma sheet, while tailward of the reconnection region field lines are open and connect to the interplanetary medium. Such continual merging on the low-latitude dawnside of the tail could supply the MPL energetic electrons observed with Imp 8. Furthermore, the peak seen in the $\left\langle C_{p}\right\rangle$ versus $\theta$ plot (Figure $11)$, which was tentatively ascribed to being near a source region, could be explained on the basis of this local acceleration model suggested for the dawn flank of the tail.

It should be noted that there is a well-known dawn-dusk asymmetry of the absolute intensity of energetic electrons within the plasma sheet [see Montgomery, 1968] with higher fluxes typically being observed along the dawnside of the tail. Continual dawnside plasma heating and energetic electron acceleration may therefore conveniently explain both the lowlatitude peak shown above in Figure 11 and the plasma sheet intensity asymmetry.

An alternative point of view to a boundary layer source might be that energetic electrons are accelerated within the plasma sheet and subsequently leak out onto magnetosheath field lines adjacent to the low-latitude dawn magnetopause, hence populating the MPL. It has been shown [e.g., Baker and Stone, 1977a] that plasma sheet electron intensities $(E \gtrsim 200$ $\mathrm{keV})$ vary between $\sim 10^{-1}$ and $\sim 10^{4}\left(\mathrm{~cm}^{2} \mathrm{~s} \mathrm{sr}\right)^{-1}$. Likewise, the observed plasma sheet electron energy spectra may be characterized by spectral indices ranging from $\gamma \sim 2$ to $\gamma \sim 7$. Electron fluxes in the plasma sheet are typically nearly isotropic, with strong streaming being only occasionally observed. Detailed analysis, probably using two or more spacecraft, would be required to establish whether plasma sheet energetic electron intensities are always sufficiently high at any given time to be supplying the tailward MPL. Figures 4, 5, and 6 above reveal that a sampling of the MPL and the plasma sheet, admittedly several hours apart in time, may often show plasma sheet fluxes to be considerably lower than those in the MPL.

In summary, among the requirements for a plasma sheet leakage source would be (1) that absolute intensities of energetic electrons in the plasma sheet always be high enough to supply the persistently observed and remarkably constant average fluxes of the MPL, (2) that in leaking out of the plasma sheet, the electrons show the hard and also remarkably constant $E^{-3}$ energy spectrùm, (3) that the leakage take place adequately far forward $\left(X_{\mathrm{SM}} \gtrsim-10 R_{E}\right)$ so as to be consistent with the apparent region from which the MPL flow emanates (cf. flow vectors in Figure 3), and (4) that in order to populate the entire layer with reasonable uniformity, the electrons would have to diffuse several earth radii across the magnetosheath field lines in a time short in comparison with the escape time of the electrons along the field lines immediately adjacent to the source. Similar requirements would exist for leakage from the dayside magnetosphere.

Direct dumping of trapped particles onto recently 'opened' field lines is also a possible source. However, relativistic electrons are lost rapidly from a newly merged field line, so that a nearly continuous flow could not be maintained during the several minutes necessary for the field lines to convect tailward $\sim 30 R_{E}$.

Another possible explanation for the source of the energetic electron layer would be stochastic acceleration associated with dayside magnetic merging. This possibility has been discussed briefly in the paper by Baker and Stone [1977c], where it was shown that the energy flows within the layer are consistent with the theoretical expectations inherent in dayside (nose) merging models. This possibility will be further examined in a forthcoming paper in which we explore the relationship of the energy flow to various characteristics of the interplanetary medium. 
Acknowledgments. We thank L. A. Frank both for providing us with Imp 8 Lepedea energy-time spectograms and for many helpful discussions. We are also grateful to R. P. Lepping and N. F. Ness for providing us with the $\operatorname{Imp} 8$ magnetometer data through J. H. King of the National Space Science Data Center. We are grateful to R. E. Vogt, who has been closely associated with this investigation, and to W. E. Althouse, G. J. Hurford, J. E. Lupton, and R. A. Mewaldt, who made significant contributions to various phases of this program. This work was supported in part by the National Aeronautics and Space Administration under contract NAS5-11066 and grant NGR 05-002160.

The Editor thanks V. Domingo and another referee for their assistance in evaluating this paper.

\section{REFERENCES}

Baker, D. N., and E. C. Stone, Observations of energetic electrons ( $E$ $\gtrsim 200 \mathrm{keV}$ ) in the earth's magnetotail: Plasma sheet and fireball observations, J. Geophys. Res., 82, 1532, 1977a.

Baker, D. N., and E. C. Stone; The magnetopause electron layer along the distant magnetotail, Geophys. Res. Lett., 4, 133, $1977 b$.

Baker, D. N., and E. C. Stone, The relationship of energy flow at the magnetopause to geomagnetic activity, Geophys. Res. Lett., 4, 395, $1977 c$

Behannon, K. W., Mapping of the earth's bow shock and magnetic tail by Explorer 33, J. Geophys. Res., 73, 907, 1968.

Domingo, V., D. E. Page, and K.-P. Wenzel, Energetic electrons at the magnetopause, in Correlated Interplanetary and Magnetospheric Observations, edited by D. E. Page, p. 159, D. Reidel, Hingham, Mass., 1974.
Domingo, V., D. E. Page, and K.-P. Wenzel, Energetic and relativistic electrons near the polar magnetopause, I. Geophys. Res., 82, 2327, 1977.

Fairfield, D. H., Magnetic fields at the magnetosheath, Rev. Geophys. Space Phys., 14, 117, 1976.

Frank, L. A., and J. A. Van Allen, Measurement of energetic electrons in the vicinity of the sunward magnetospheric boundary with Explorer 14, J. Geophys. Res., 69, 4923, 1964.

Frank, L. A., K. L. Ackerson, and R. P. Lepping, On hot tenuous plasmas, fireballs, and boundary layers in the earth's magnetotail, $J$. Geophys. Res., 81, 5859, 1976.

Meng, C.-I., and K. A. Anderson, A layer of energetic electrons $(E>$ $40 \mathrm{keV}$ ) near the magnetopause, J. Geophys. Res., 75, 1827, 1970.

Meng, C.-I., and K. A. Anderson, Characteristics of the magnetopause energetic electron layer, J. Geophys. Res., 80, 4237, 1975.

Montgomery, M. D., Observations of electrons in the earth's magnetotail by Vela 2, J. Geophys. Res., 73, 871, 1968.

Page, D. E., V. Domingo, D. Köhn, B. G. Taylor, K.-P. Wenzel, and P. C. Hedgecock, High-energy electrons at the magnetopause above the north pole, Space Res., 13, 631, 1973.

Singer, S., and S. J. Bame, Anisotropic distributions of energetic electrons in the earth's magnetotail and magnetosheath, in Particles and Fields in the Magnetosphere, edited by B. M. McCormac, p. 122, D. Reidel, Hingham, Mass., 1970.

(Received December 6, 1977; revised January 24, 1978; accepted April 3, 1978.) 